\title{
Equilibrium Traffic Flow Assignment in Case of Two Navigation Providers
}

\author{
Victor Zakharov $^{1,2}$, Alexander Krylatov ${ }^{1,2}$, and Dmitry Ivanov ${ }^{3}$ \\ ${ }^{1}$ Saint-Petersburg State University, 199034 Saint-Petersburg, Russia \\ mcvictor@mail.ru \\ ${ }^{2}$ Institute of Transportation Problem of Russian Academy of Sciences \\ mcvictor@mail.ru, partisan-sasha@yandex.ru \\ ${ }^{3}$ Berlin School of Economics and Law, 10825 Berlin, Germany \\ divanov@hwr-berlin.de
}

\begin{abstract}
Traffic flow assignment system that includes two navigation providers is considered. Navigation providers predict future traffic conditions and provide travel guidance for given original-destination demand and network capacity. Three classes of drivers are introduced. The users of navigation service from the first and the second classes are supplied with the shortest path guidance by navigation providers «Navigator 1 » and «Navigator 2 » correspondingly. The third class of drivers is characterized by user-optimal behavior without current guidance information about traffic conditions. The main goals of Navigators are to assign the traffic flow of their customers (users) so as to maximize appropriate pay-off functions which depend on original estimation of traffic volume delay and are influenced by decision of both of them. Existence and uniqueness of Nash equilibrium in considered non-zero sum game are proven and an explicit form of Nash equilibrium is presented.
\end{abstract}

Keywords: Traffic flow assignment, routing, dynamics, Nash equilibrium.

\section{Introduction}

The impact of information technologies (IT) on transportation processes becomes more and more crucial. Most of the new technologies share attributes of intelligence. Cyber-physical systems incorporate elements from both information and material (physical) subsystems and processes which are integrated and decisions in them are cohesive [1]. Elements of physical processes are supported by information services. Cyber-physical systems are characterized by decentralization and the autonomous behavior of their elements.

We consider three groups of customers (drivers on the streets): two groups which are guided by different providers of navigation and one group which do not use any navigation system. The study is focused on the existence and analytical representation of strategies of navigation providers which form Nash equilibrium. Providers are considered as non-collaborative players in non-cooperative game. They are interesting in higher level of service for their customers which would attract them to buy 
navigation system. We assume that using fast and efficient algorithms for calculating routs upon request of a user to minimize travel time from origin to destination leads to increasing level of service. Therefore designing the fast algorithms for assignment vehicle is crucial for both theory and practice [2]. The study of a navigator's strategic behavior in traffic flow assignment is important because a lot of drivers in megalopolis can use routes recommended by navigation systems (navigators) to reach their destinations with minimum driving time.

\section{State of the Art}

Determining the equilibrium traffic flow assignment has been a preoccupation of transport planners for nearly half a century. J.G. Wardrop put forth the definition of the steady state equilibrium of a traffic network in 1952 [3]. Beckmann et al. in 1956 provided an analytical construct of Wardrop's verbal criteria [4]. Within a relatively short period, between 1968 and 1973, several algorithms were identified, analysed and solved computationally [5]. In addition, the linearization method based on the Frank and Wolfe algorithm emerged in 1956 [6].

Since the early 1990s, game theoretic models have been employed in the context of traffic flow assignment and control [7, 8], routing [9] and virtual path bandwidth allocation in modern networking. Then, using game theory framework in routing optimization was continued in the second part of the 1990s [8, 10,11].

In the first decade of XXI century equilibrium resource assignment models have started to emerge in a field that is quite close to the transportation system - in telecommunication systems $[12,13]$. In this way, the results from traffic flow assignment can be used in telecommunication systems and vice versa. That is why in this paper we will follow the results of studies by Altman et al. [14, 15]. We interpret transmitters from wireless networks as providers of navigation (navigators) in traffic flow networks and consider the traffic flow assignment problem as a non-zero-sum game. Pay-off functions are used in the proposed model close to those of Altman et al. [14, 15].

The novelty of the present paper is primarily in the use of a game theory framework and special pay-offs in a game of navigation providers. This study proposes a method for fast calculation Nash equilibrium in a corresponding game of two navigators (players) due to explicit form of the solution. The results might be highly applicable in the sense that computations allow the finding of optimal routes for customers of navigation providers.

\section{Traffic Assignment Game of Two Navigation Providers}

Consider a traffic flow assignment system which includes two navigation providers who predicts future traffic conditions and provide travel guidance given originaldestination demand and network capacity. Suppose there are three classes of users. The first and second class are supplied with the shortest path guidance by navigation providers «Navigator $1 »$ and «Navigator 2» correspondingly and the users also believe that guidance information is $100 \%$ accurate. The third class is characterized by user-optimal behavior without current guidance information about traffic conditions. 
Transportation network is presented as a directed graph with two nodes (origin and destination) and $n$ arcs (paths) going from origin to destination. The main goals of Navigators are to assign the traffic flow of their customers (users) from origin to destination through $n$ paths so as to maximize their appropriate pay-off functions which influenced by decision of both of them. Due to this influence we can consider a noncooperative game of two players.

Let's introduce the following notations:

$i$ - index number of the path, $i \in\{1, n\}$;

$j-$ index number of Navigator, $j \in\{1,2\}$;

$F^{j}>0$ - traffic flow volume (quantity of customers) assigned by Navigator $j$;

$f_{i}^{j} \geq 0-$ traffic flow volume that Navigator $j$ sends on $i$-th path;

$h_{i} \geq 0-$ traffic flow of the users from third class on path $i, H=\sum_{i=1}^{n} h_{i}$;

$g_{i}>0-$ capacity of $i$-th path;

$d_{i}^{j}>0-$ traffic flow level of Navigator $j$ customers delay on path $i$.

Suppose the total volume of traffic is less then total capacities of paths $H+F^{1}+F^{2} \leq \sum_{i=1}^{n} g_{i}$. Define set of strategies of player $j$ as set of vectors $f^{j}=\left(f_{1}^{j}, \ldots, f_{n}^{j}\right)$ such that

$$
\sum_{i=1}^{n} f_{i}^{j}=F^{j}
$$

We assume that each Navigator is able to estimate the level of congestion on each path of transportation network for given strategy of another player and allocation $h=\left(h_{1}, \ldots, h_{n}\right)$ of users of the third class. We also assume that the level of congestion for customers of Navigator 1 on $i$-th path is increasing with respect to sum of total amount of customers $f_{i}^{2}$ of Navigator 2 sent to the $i$-th path and traffic flow volume of customers $h_{i}$ divided by capacity of $i$-th path. Thus we suppose that delay for each customer of Navigation 1 following path $i$ may be estimated as follows

$$
d_{i}^{1}=\frac{f_{i}^{2}+h_{i}}{g_{i}}, \forall i=\overline{1, n}
$$

The same estimation may be applied for delay for each customer of Navigator 2 given strategy of player 1 and allocation $h=\left(h_{1}, \ldots, h_{n}\right)$ of users of the third class:

$$
d_{i}^{2}=\frac{f_{i}^{1}+h_{i}}{g_{i}}, \forall i=\overline{1, n}
$$


For estimation of the pay-offs of Navigator $j$, the following utility function is considered that quantifies a trade-off between throughput and delay [16]

$$
V_{j}\left(f^{1}, f^{2}\right)=\frac{\left(\sum_{i=1}^{n} f_{i}^{j}\right)^{\beta}}{\operatorname{del}_{j}\left(f^{1}, f^{2}\right)}
$$

where $\operatorname{del}_{j}\left(f^{1}, f^{2}\right)$ is the function that characterizes the average delay experienced by the Navigator $j$ and $\beta$ is a trade-off parameter. Such a utility function is commonly used in the literature in applications that are sensitive to throughput as well as delay [17]. It consists of the ratio between the expected throughput and the expected delay. Thus it captures preferences towards higher throughputs and penalizes large delays. One of disadvantages of this expression is using average delay instead of vector of delay values on each of the paths.

We propose to introduce explicit form of pay-off functions to be maximized by the players which takes into account expressions (2) and (3). Assume that pay-offs are linear combination of corresponding volumes of flows to each of paths weighted with inverse values of delays

$$
\begin{aligned}
& V_{1}\left(f^{1}, f^{2}\right)=\sum_{i=1}^{n} \frac{1}{d_{i}^{1}} f_{i}^{1} \\
& V_{2}\left(f^{1}, f^{2}\right)=\sum_{i=1}^{n} \frac{1}{d_{i}^{2}} f_{i}^{2}
\end{aligned}
$$

We can see that to maximize pay-off player has to decrease the flow sent to the path with higher delay therefore. Thus it leads to less travel time along this path. Substituting (2) to (4) and (3) to (5) we are getting expressions for pay-off functions of the players as follows

$$
\begin{aligned}
& V_{1}\left(f^{1}, f^{2}\right)=\sum_{i=1}^{n} \frac{g_{i} f_{i}^{1}}{f_{i}^{2}+h_{i}} \\
& V_{2}\left(f^{1}, f^{2}\right)=\sum_{i=1}^{n} \frac{g_{i} f_{i}^{2}}{f_{i}^{1}+h_{i}}
\end{aligned}
$$

In these settings, Navigators simultaneously try to assign flow of their customers through more appropriate ways using information about capacity of paths and «base» flows of drivers from third class.

By definition strategies $f^{1^{*}}$ and $f^{2^{*}}$ form Nash equilibrium situation, if for any strategies admissible strategies $f^{1}$ and $f^{2}$ the following inequalities hold:

$$
v_{1}\left(f^{1}, f^{2^{*}}\right) \leq v_{1}\left(f^{1^{*}}, f^{2^{*}}\right)
$$




$$
V_{2}\left(f^{1^{*}}, f^{2}\right) \leq V_{2}\left(f^{1^{*}}, f^{2^{*}}\right)
$$

Since functions (6) and (7) are concave in $f^{1}$ and $f^{2}$ respectively, the Kuhn - Tucker conditions imply the following theorem.

Theorem 1. $\left(f^{1^{*}}, f^{2^{*}}\right)$ is a Nash equilibrium if and only if there are non-negative $\omega^{1}$ and $\omega^{2}$ (Lagrange multipliers) such that

$$
\begin{gathered}
\frac{g_{i}}{f_{i}^{2}+h_{i}}\left\{\begin{array}{l}
=\omega^{1} \text { for } f_{i}^{1^{*}}>0 \\
\leq \omega^{1} \text { for } f_{i}^{1^{*}}=0
\end{array}\right. \\
\frac{g_{i}}{f_{i}^{1}+h_{i}}\left\{\begin{array}{l}
=\omega^{2} \text { for } f_{i}^{2^{*}}>0 \\
\leq \omega^{2} \text { for } f_{i}^{2^{*}}=0
\end{array}\right.
\end{gathered}
$$

where $i=\overline{1, n}$.

Corollary. Each Nash equilibrium is of the form $\left(f^{1}\left(\omega^{1}, \omega^{2}\right), f^{2}\left(\omega^{1}, \omega^{2}\right)\right)$ for some positive $\omega^{1}$ and $\omega^{2}$.

In order to find the equilibrium strategies, find $\omega^{1}$ and $\omega^{2}$ such that the following conditions hold

$$
Q^{j}\left(\omega^{1}, \omega^{2}\right)=\sum_{i=1}^{n} f_{i}^{j}\left(\omega^{1}, \omega^{2}\right)=F^{j}
$$

Due to the properties of payoff-functions the following lemma can be proved.

Lemma. The system of non-linear equations (11) has a unique positive solution $\left(\omega^{1^{*}}, \omega^{2^{*}}\right)$.

Due to Theorem 1 and Lemma, it becomes possible to obtain the explicit form of optimal strategies for Navigators in the game of traffic flow assignment. For this purpose, optimal values of $\omega^{1}$ and $\omega^{2}$ have to be found from the equations: $\sum_{\omega^{2}<\frac{g_{i}}{h_{i}}}\left(\frac{g_{i}}{\omega^{2}}-h_{i}\right)=F^{1}$ and $\sum_{\omega^{1}<\frac{g_{i}}{h_{i}}}\left(\frac{g_{i}}{\omega^{1}}-h_{i}\right)=F^{2}$.

To find the solution in an analytical form, we have to assume that there not exist such $s, I=\{1, n\}$ that $\frac{h_{s}}{g_{s}}=\frac{h_{l}}{g_{l}}$. Then without loss of generality, it can be assumed that

$$
\frac{h_{1}}{g_{1}}<\frac{h_{2}}{g_{2}}<\ldots<\frac{h_{n}}{g_{n}}
$$

In the performance of these conditions the following Theorem 2 has appeared. 
Theorem 2. Nash equilibrium in a game of two Navigators with pay-offs (6) and (7) is achieved by the following strategies using

$$
\begin{aligned}
& f_{i}^{1^{*}}=\left\{\begin{array}{l}
\frac{g_{i} F^{1}+g_{i} \sum_{r=1}^{k_{1}} h_{r}}{\sum_{r=1}^{k_{1}} g_{r}}-h_{i}, \text { if } i \leq k_{1} \\
0, \quad \text { if } i>k_{1}
\end{array}\right. \\
& f_{i}^{2^{*}}=\left\{\begin{array}{l}
\frac{g_{i} F^{2}+g_{i} \sum_{r=1}^{k_{2}} h_{r}}{\sum_{r=1}^{k_{2}} g_{r}}-h_{i}, \text { if } i \leq k_{2} \\
0, \quad \text { if } i>k_{2}
\end{array}\right.
\end{aligned}
$$

where $k_{1}$ and $k_{2}$ can be found from the following conditions:

$$
\varphi_{k_{j}}<F^{j} \leq \varphi_{k_{j}+1}
$$

where

$$
\varphi_{t}=\sum_{i=1}^{t} g_{i}\left(\frac{h_{t}}{g_{t}}-\frac{h_{i}}{g_{i}}\right) \quad \text { for } \quad t \in\{1, n\}
$$

and $k_{j}=n$ in case $F^{j}>\varphi_{n}$.

Results of corollary, lemma and theorem 2 allows us to formulate the following theorem.

Theorem 3. A game of two navigation providers in the performance of (12) has unique Nash equilibrium $\left(f^{1^{*}}, f^{2^{*}}\right)$ which can be found from (13) - (16).

\section{Conclusion}

In a megalopolis, many drivers can use routes provided by different navigation systems (navigators) to reach their destinations with minimum driving time. Therefore, navigators are interested in providing their customers with a higher level of service compare to that of other competitors. The novelty of the present paper is primarily in the use of a game theory framework and special pay-offs in a game of navigation providers. This study proposes a method for fast calculation Nash equilibrium in a corresponding game of two navigators (players) due to explicit form of the solution. The results might be highly applicable in the sense that computations allow fast finding optimal routes for customers of navigation providers. The explicit form of Nash equilibrium has been demonstrated. 


\section{References}

1. Zhuge, H.: Semantic linking through spaces for cyber-physical-socio intelligence: A methodology. Artif. Intell. 175(5-6), 988-1019 (2011)

2. Kerner, B.S.: Introduction to Modern Traffic Flow Theory and Control: The Long Road to Three-Phase Traffic Theory. Springer, Berlin (2009)

3. Wardrop, J.G.: Some theoretical aspects of road traffic research. Proc. Institution of Civil Engineers II, 325-378 (1952)

4. Beckmann, M.J., McGuire, C.B., Winsten, C.B.: Studies in the Economics of Transportation. Yale University Press, New Haven (1956)

5. Florian, M., Nguyen, S., Ferland, J.: On the combined distribution-assignment of traffic. Transportation Science 9, 43-53 (1975)

6. Frank, M., Wolfe, P.: An algorithm for quadratic programming. Naval Research Logistics Quarterly 3, 95-110 (1956)

7. Altman, E.: Flow control using the theory of zero-sum markov games. IEEE Transaction on Automatic Control 39, 814-818 (1994)

8. Korilis, Y.A., Lazar, A.A., Orda, A.: Architecting noncooperative networks. IEEE Journal on Selected Areas in Communications 15(7), 1241-1251 (1995)

9. Orda, A., Rom, R., Shimkin, N.: Competitive routing in multiuser communication networks. IEEE/ACM Transactions on Networking 1(5), 510-521 (1993)

10. Korilis, Y.A., Lazar, A.A., Orda, A.: Achieving network optima using Stackelberg routing strategies. Networking, IEEE/ACM Transactions on Networking 5(1), 161-173 (1997)

11. Korilis, Y.A., Lazar, A.A., Orda, A.: Capacity allocation under noncooperative routing. IEEE Transaction on Automatic Control 42(3), 309-325 (1997)

12. Altman, E., Wynter, L.: Equilibrium, Games, and Pricing in Transportation and Telecommunication Networks. Networks and Spatial Economics 4, 7-21 (2004)

13. Lasaulce, S., Tembine, H.: Game Theory and Learning for Wireless Networks: Fundamentals and Applications. Elsevier, New York (2011)

14. Altman, E., Avrachenkov, K., Garnaev, A.: Jamming Game in Wireless Networks with Transmission Cost. In: Chahed, T., Tuffin, B. (eds.) NET-COOP 2007. LNCS, vol. 4465, pp. 1-12. Springer, Heidelberg (2007)

15. Altman, E., Avrachenkov, K., Garnaev, A.: Closed Form Solutions for Water-Filling Problem in Optimization and Game Frameworks. Telecommunication Systems Journal 47, 153-164 (2009)

16. Altman, E., Basar, T., Srikant, R.: Nash equilibria for combined flow control and routing in networks: asymptotic behavior for a large number of users. IEEE Transactions on Automatic Control 47(6), 917-930 (2002)

17. Ching, W.K.: A note on the convergence of asynchronous greedy algorithm with relaxation in a multiclass queuing environment. IEEE Commun. Lett. 3, 34-36 (1999)

\section{Appendix}

Proof of Theorem 1. We proof this theorem for Navigator 1. For Navigator 2 the proof is analogical.

Function $v_{1}$ is linear with respect to components $f^{1}$, that means it is both convex and concave. Such a fact allows us to apply the Kuhn-Tucker theorem. The Lagrangian corresponding to minimization of $-V_{1}$ subject to the constraints (1) and nonnegativity constraints on $f_{i}^{1}$ is given by 


$$
L^{1}=-\sum_{k=1}^{n} \frac{g_{k} f_{k}^{1}}{f_{k}^{2}+h_{k}}+\omega^{1}\left(\sum_{k=1}^{n} f_{k}^{1}-F^{1}\right)+\sum_{k=1}^{n} \eta_{k}^{1}\left(-f_{k}^{1}\right)
$$

In differentiating the Lagrangian with respect to $f_{i}^{1}$ and equating the derivative to zero, we obtain (stationarity)

$$
\omega^{1}=\frac{g_{i}}{f_{i}^{2}+h_{i}}+\eta_{i}^{1}
$$

Lagrangian multipliers are non-negative due to non-negativity of $g_{i}, f_{i}^{1}$ and $h_{i}$. Now, let us employ complementary slackness condition $\eta_{i}^{1} f_{i}^{1}=0$. Such an equation means that at least one of the multipliers is equal to zero. Therefore, in case $f_{i}^{1}>0$ it is necessary $\eta_{i}^{1}=0$ and we obtain the first part of expression (9). In case $f_{i}^{1}=0$ the value of $\eta_{i}^{1} \geq 0$ and we obtain the second part of expression (9).

Proof of Theorem 2. We prove the theorem for Navigator 1. For Navigator 2 the proof is absolutely analogous.

First note $\hat{Q}^{1}\left(\omega^{2}\right)=0$ for $\omega^{2} \leq T$ where $T=\max _{i \in\{1, n\}} \frac{g_{i}}{h_{i}}$ as above, $\hat{Q}^{1}\left(\omega^{2}\right)$ is strictly positive and decreasing in $(0, T)$. Let $k_{1} \in\{1, n\}$ be such that $\frac{g_{k_{1}}}{h_{k_{1}}}>\omega^{2} \geq \frac{g_{k_{1}+1}}{h_{k_{1}+1}}$ where $\frac{g_{i}}{h_{i}}=0$ when $i=n+1$. Then, $\left[\frac{g_{i}}{\omega^{2}}-h_{i}\right]_{+}=\frac{g_{i}}{\omega^{2}}-h_{i}$ for $i \in\left\{1, k_{1}\right\}$ and $\left[\frac{g_{i}}{\omega^{2}}-h_{i}\right]_{+}=0$ for $i \in\left\{k_{1}+1, n\right\}$. So, $\hat{Q}^{1}\left(\omega^{2}\right)=\sum_{i=1}^{k_{1}}\left(\frac{g_{i}}{\omega^{2}}-h_{i}\right)$. Since $\hat{Q}^{1}\left(\omega^{2^{*}}\right)=F^{1}$ we have that $\omega^{2^{*}}=\frac{\sum_{r=1}^{k_{1}} g_{r}}{F^{1}+\sum_{r=1}^{k_{1}} h_{r}}$. Because $\hat{Q}^{1}\left(\omega^{2}\right)$ is strictly decreasing on $(0, T)$ we can find $k_{1}$ from the following condition $\widehat{Q}^{1}\left(\frac{g_{k_{1}}}{h_{k_{1}}}\right)<F^{1} \leq \widehat{Q}^{1}\left(\frac{g_{k_{1}+1}}{h_{k_{1}+1}}\right)$.

Since $\sum_{i=1}^{k_{1}}\left(g_{i} \frac{h_{k_{1}}}{g_{k_{1}}}-h_{i}\right)=\sum_{i=1}^{k_{1}+1}\left(g_{i} \frac{h_{k_{1}+1}}{g_{k_{1}+1}}-h_{i}\right)$ the integer $k_{1}$ can be found from the following equivalent condition

$$
\varphi_{k_{1}}<F^{1} \leq \varphi_{k_{1}+1}
$$

Therefore, the two last results imply Theorem 2 . 